\title{
On the number of polynomials of bounded measure
}

by

\section{A. Dubickas (Vilnius) and S. V. Konyagin (Moscow)}

1. Introduction. The Mahler measure of the polynomial

$$
f(x)=a_{0} x^{d}+a_{1} x^{d-1}+\ldots+a_{d}=a_{0}\left(x-\alpha_{1}\right) \ldots\left(x-\alpha_{d}\right), \quad a_{0} \neq 0,
$$

is defined by

$$
M(f)=M(f(x))=\left|a_{0}\right| \prod_{j=1}^{d} \max \left(1,\left|\alpha_{j}\right|\right) .
$$

Our purpose here is to give an upper bound for the cardinality of the set of polynomials in $\mathbb{Z}[x]$ of given degree and of bounded Mahler's measure. Since $M\left( \pm x^{t} f(x)\right)=M(f(x))$, let us suppose that $a_{0}>0$ and $a_{d} \neq 0$. This allows us to avoid some trivial complications. We will denote by $N(d, T)$ the set of polynomials $f(x) \in \mathbb{Z}[x]$ of degree $d$ such that $M(f) \leq T, a_{0}>0$, $f(0)=a_{d} \neq 0$.

Firstly, notice that if $T<1$ then the set $N(d, T)$ is empty. Secondly, if $T=1$ then by Kronecker's theorem each $f(x)$ in $N(d, 1)$ is a product of cyclotomic polynomials. The result of E. Dobrowolski [Do] implies that $N(d, T)$ is of the same structure for $T<\exp \left(c_{1}\left(\frac{\log \log d}{\log d}\right)^{3}\right)$. For a sufficiently large $d$ this holds with $c_{1}=9 / 4-\varepsilon, \varepsilon>0$ [Lo]. By Lehmer's conjecture [Le] there exists a positive constant $\delta$ such that the set $N(d, T)$ has the same structure for $T<1+\delta$. Therefore for small $T$ the cardinality of $N(d, T)$ is equal to the number of ways to write $d$ as a sum of the form

$$
d=\sum_{m \geq 1} d_{m} \varphi(m)
$$

where $\varphi$ is the Euler totient function and $d_{m}$ is a nonnegative integer. The asymptotics for this number was found by D. W. Boyd and H. L. Montgomery [Bo-Mo].

The structure of the set $N(d, T)$ for larger $T$ is much more complicated. Let us start by giving some trivial lower and upper bounds for card $N(d, T)$.

1991 Mathematics Subject Classification: 11C08, 11R04, 11R09, 12D10. 
By Landau's inequality [La],

$$
M(f) \leq \sqrt{\sum_{j=0}^{d} a_{j}^{2}} \leq \sqrt{d+1} \max _{0 \leq j \leq d}\left|a_{j}\right| .
$$

Hence all polynomials with integer coefficients such that $1 \leq a_{0} \leq T / \sqrt{d+1}$, $\left|a_{j}\right| \leq T / \sqrt{d+1}, j=1, \ldots, d, a_{d} \neq 0$ lie in $N(d, T)$. If $T \geq \sqrt{d+1}$, we can bound card $N(d, T)$ from below by

$$
\left[\frac{T}{\sqrt{d+1}}\right]\left(2\left[\frac{T}{\sqrt{d+1}}\right]+1\right)^{d-1} 2\left[\frac{T}{\sqrt{d+1}}\right]>\frac{1}{2}\left(\frac{T}{\sqrt{d+1}}\right)^{d+1} .
$$

Here and throughout the paper [ ] denotes the integral part. If $1 \leq T<$ $\sqrt{d+1}$, then this lower bound holds trivially. Hence we obtain the following lower bound:

$$
\operatorname{card} N(d, T)>\frac{1}{2} T^{d+1}(d+1)^{-(d+1) / 2} .
$$

On the other hand, if $M(f) \leq T$ then

$$
\left|a_{j}\right|=\left|a_{0}\right| \cdot\left|\alpha_{1} \alpha_{2} \ldots \alpha_{j}+\ldots+\alpha_{d-j+1} \ldots \alpha_{d}\right| \leq\left(\begin{array}{l}
d \\
j
\end{array}\right) T .
$$

Hence card $N(d, T)$ is bounded from above by

$$
\begin{aligned}
2[T]^{2} \prod_{j=1}^{d-1}\left(2\left[\left(\begin{array}{l}
d \\
j
\end{array}\right) T\right]+1\right) & \leq 2 T^{2} \prod_{j=1}^{d-1}\left(2\left(\begin{array}{l}
d \\
j
\end{array}\right) T+1\right) \\
& \leq 2 \cdot 3^{d-1} T^{d+1} \prod_{j=1}^{d-1}\left(\begin{array}{l}
d \\
j
\end{array}\right) \\
& =2 \cdot 3^{d-1} T^{d+1} d !^{-d-1} \prod_{j=1}^{d} j^{2 j}
\end{aligned}
$$

It is an easy exercise to show by induction that

$$
\prod_{j=1}^{d} j^{2 j} \leq d^{d(d+1)} \exp \left(\frac{d-d^{2}}{2}\right) .
$$

Utilizing Stirling's formula

$$
d !>\sqrt{2 \pi d}\left(\frac{d}{e}\right)^{d} \exp \left(-\frac{1}{12 d}\right),
$$

we get 


$$
\begin{aligned}
\operatorname{card} N(d, T)< & T^{d+1} \exp \left(\frac{d^{2}}{2}+\frac{3 d}{2}\right. \\
& \left.+\frac{d+1}{12 d}+(d-1) \log 3+\log 2-\frac{d+1}{2} \log (2 \pi d)\right) .
\end{aligned}
$$

Thus, for sufficiently large $d$,

$$
\operatorname{card} N(d, T)<T^{d+1} \exp \left(d^{2} / 2\right) .
$$

Comparing these trivial estimates (1) and (2) we see that they differ by a factor depending on $d$ only. So for "large" $T$ we cannot expect to do much better than (2). However, for "small" $T$, e.g. fixed $T$, this bound can be substantially improved. For example, the interesting case $T<2$ was considered by M. Mignotte [Mi]. He proved that the number of irreducible polynomials of degree $d$ and of Mahler's measure smaller than 2 is less than $2(8 d)^{2 d+1}$. Combined with the results of [Bo-Mo] this implies that

$$
\operatorname{card} N(d, T)<d^{c_{2} d}
$$

for $T<2$ and some absolute positive constant $c_{2}$.

Denote by $N_{1}(d, T)$ the set of monic $\left(a_{0}=1\right)$, irreducible polynomials $f(x) \in \mathbb{Z}[x]$ of degree $d$ such that $M(f) \leq T,|f(0)|=\left|a_{d}\right|=1$. Recently one of the authors $[\mathrm{Ko}]$ proved that

$$
\operatorname{card} N_{1}(d, T)<T^{c_{3} d} \exp \left(d^{19 / 20}\right)
$$

provided that $d$ is sufficiently large where $c_{3}$ is an effectively computable constant.

In all what follows, let $d$ be a sufficiently large positive integer. Throughout this paper $c, c_{4}, c_{5}, c_{6}, \ldots$ will be assumed to be positive effective constants, and $\varepsilon, \varepsilon_{1}, \varepsilon_{2}$ will be assumed to be small positive constants. Let $\theta=1.32471 \ldots$ be the real root of the polynomial $x^{3}-x-1$.

Theorem. (i) If $1 \leq T<\exp \left(\left(\frac{9}{4}-\varepsilon\right)\left(\frac{\log \log d}{\log d}\right)^{3}\right)$, then

$$
\operatorname{card} N(d, T)=c_{4}(\log d)^{-1 / 2} d^{-1} \exp \left(\frac{1}{\pi} \sqrt{105 \zeta(3) d}\right)(1+o(1)),
$$

where $c_{4}=\frac{1}{4 \pi^{2}}\left(105 \zeta(3) e^{-\gamma}\right)^{1 / 2}$ and $o(1) \rightarrow 0$ as $d \rightarrow \infty$.

(ii) If $\exp \left(\left(\frac{9}{4}-\varepsilon\right)\left(\frac{\log \log d}{\log d}\right)^{3}\right) \leq T<\theta$, then

$$
\operatorname{card} N(d, T)<T^{d(1 / 2+c \log \log d / \log d)} .
$$

(iii) If $\theta \leq T$, then

$$
\operatorname{card} N(d, T)<T^{d(1+c \log \log d / \log d)} .
$$

We will prove (ii) and (iii) with $c=16$. The case (i) is given here only for completeness. As mentioned above it is a combination of the results 
obtained in [Lo] and [Bo-Mo]. Combining (i)-(iii) we obtain the following general estimate for every positive $\varepsilon_{1}$ :

$$
\begin{aligned}
\operatorname{card} N(d, T) & <T^{d(1+16 \log \log d / \log d)} \exp (3.58 \sqrt{d}) \\
& <T^{\left(1+\varepsilon_{1}\right) d} \exp (4 \sqrt{d}) .
\end{aligned}
$$

Taking $T=2$ in (5) we obtain card $N(d, 2)<\left(2+\varepsilon_{2}\right)^{d}$, which strengthens the inequality (3). Notice also that (5) strengthens (4), since card $N(d, T) \geq$ card $N_{1}(d, T)$. However, for large $T, T>\exp \left(\frac{d \log d}{2 c \log \log d}\right)$, the trivial inequality (2) gives a stronger upper bound than (iii).

In Section 2 we give some auxiliary lemmas. Section 3 contains a sketch of proof. The proof of (iii) is given in Sections 4 and 5 where we bound the number of polynomials with small and large leading coefficients respectively. Finally, in Section 6 we complete the proof of (ii).

The authors thank the organizers of the Number Theory Conference dedicated to Professor A. Schinzel in Zakopane (July 1997). In the pleasant atmosphere of this conference the idea to write this paper came and a part of the work was done. The research of the second named author was supported by Grants 96-01-00378 and 96-15-96072 from the Russian Foundation for Basic Research.

2. Auxiliary lemmas. Let $e_{1}, \ldots, e_{d}$ be the natural basis of the $d$ dimensional Euclidean space $\mathbb{R}^{d}$. We put

$$
\|x\|=\max _{1 \leq j \leq d}\left|x_{j}\right|
$$

for the $l_{\infty}$ norm of the vector $x=x_{1} e_{1}+\ldots+x_{d} e_{d}$. For a convex closed bounded set $A \subset \mathbb{R}^{d}$ and $j=1, \ldots, d$ we put

$$
F_{j}(A)=A+\left[-e_{j} / 2 ; e_{j} / 2\right]=\left\{x+\mu e_{j}: x \in A,|\mu| \leq 1 / 2\right\} .
$$

Let $F(A)$ be the $1 / 2$-neighbourhood of $A$ :

$$
F(A)=\{x+y: x \in A,\|y\| \leq 1 / 2\}=F_{1}\left(F_{2} \ldots F_{d}(A) \ldots\right) .
$$

Suppose that $G \subset\{1, \ldots, d\}$ and $g=\operatorname{card} G$. We denote by $\mathcal{O}_{G}(A)$ the orthogonal projection of the set $A$ onto the linear space spanned by the vectors $e_{j}$ with $j \in G$. Finally, we denote by $\operatorname{Vol}_{g} \mathcal{O}_{G}(A)$ the volume of the $g$-dimensional $(1 \leq g \leq d)$ convex set $\mathcal{O}_{G}(A)$, and let $\operatorname{Vol}_{0} \mathcal{O}_{\emptyset}(A)=1$. With this notation we have the following general lemma for $d \geq 1$ :

LEMma 0. We have

$$
\operatorname{Vol}_{d} F(A)=\sum \operatorname{Vol}_{g} \mathcal{O}_{G}(A),
$$

where the sum is taken over all subsets $G$ of $\{1, \ldots, d\}$. 
Proof. Let $\widehat{A}$ be a convex closed bounded set in $\mathbb{R}^{d}$, and let $G \subset$ $\{1, \ldots, d\}, j \in G$. Expressing the volumes $\mathcal{O}_{G}(\widehat{A})$ and $\mathcal{O}_{G}\left(F_{j}(\widehat{A})\right)$ as integrals over $\mathcal{O}_{G \backslash\{j\}}(\widehat{A})$ we get

$$
\begin{aligned}
\operatorname{Vol}_{g} \mathcal{O}_{G}\left(F_{j}(\widehat{A})\right)-\operatorname{Vol}_{g} \mathcal{O}_{G}(\widehat{A}) & =\left(\frac{1}{2}+\frac{1}{2}\right) \operatorname{Vol}_{g-1} \mathcal{O}_{G \backslash\{j\}}(\widehat{A}) \\
& =\operatorname{Vol}_{g-1} \mathcal{O}_{G \backslash\{j\}}(\widehat{A}) .
\end{aligned}
$$

Therefore, we obtain the following reduction formula:

$$
\operatorname{Vol}_{g} \mathcal{O}_{G}\left(F_{j}(\widehat{A})\right)=\operatorname{Vol}_{g} \mathcal{O}_{G}(\widehat{A})+\operatorname{Vol}_{g-1} \mathcal{O}_{G \backslash\{j\}}(\widehat{A}) .
$$

Note that

$$
F(A)=\mathcal{O}_{\{1, \ldots, d\}}\left(F_{1}\left(F_{2} \ldots F_{d}(A) \ldots\right)\right) .
$$

Taking into account that the operators $\mathcal{O}_{G}$ and $F_{j}$ commute for $j \in G$, we can apply the above formula when proceeding with $\mathrm{Vol}_{d} F(A)$, thus reducing one of $F_{j}$ 's at each step. It is easy to see that in this way we finally get the stated formula.

The next lemma is an estimate of the $1 / 2$-capacity [Ti, 1.1.7] of convex polytopes contained in a parallelepiped.

Lemma 1. Suppose that $0<\tau<1 / 12, d>d(\tau)$ and let

$$
P=\prod_{j=1}^{d}\left[-u_{j} / 2 ; u_{j} / 2\right]
$$

be a parallelepiped in $\mathbb{R}^{d}$. Let $A \subset P$ be a convex polytope with at most $d^{-1+1 /(10 \tau)}$ vertices. If $D$ is a subset of $A$ such that the distance between any two elements of $D$ is at least 1 , then

$$
\operatorname{card} D<\exp \left(d^{1-9 \tau}\right) \prod_{j=1}^{d}\left(1+u_{j} d^{6 \tau-1 / 2}\right) .
$$

Proof. The unit cubes with centers at the points of $D$ are mutually nonoverlapping. The union of these cubes is a subset of $F(A)$, so that card $D \leq \operatorname{Vol}_{d} F(A)$. By Lemma 0, it remains to estimate each $\operatorname{Vol}_{g} \mathcal{O}_{G}(A)$ from above.

Suppose first that $g \geq d^{1-10 \tau}$. Then $\mathcal{O}_{G}(A)$ is a convex polytope with at most

$$
d^{-1+1 /(10 \tau)} \leq g^{(-1+1 /(10 \tau)) /(1-10 \tau)}=g^{1 /(10 \tau)}
$$

vertices. Clearly, $\mathcal{O}_{G}(A)$ is contained in the parallelepiped

$$
\mathcal{O}_{G}(P)=\prod_{j \in G}\left[-u_{j} / 2 ; u_{j} / 2\right] \subset \mathbb{R}^{g} .
$$


We now need an upper bound for the volume of a convex polytope $A_{g}$ with $\leq g^{1 /(10 \tau)}$ vertices contained in a parallelepiped $\Pi_{g} \subset \mathbb{R}^{g}$. Without loss of generality we can assume that $\Pi_{g}$ is the $g$-dimensional cube $[-1 / \sqrt{g} ; 1 / \sqrt{g}]^{g}$ inscribed in the unit ball $B_{g}$. The volume of the ball is estimated by the volume of the cube as follows:

$$
\mathrm{Vol}_{g} B_{g}=\frac{\pi^{g / 2}}{\Gamma(1+g / 2)}<\frac{\pi^{g / 2}}{(g /(2 e))^{g / 2}}=\left(\frac{2 \pi e}{g}\right)^{g / 2}=(\pi e)^{g / 2} \operatorname{Vol}_{g} \Pi_{g} .
$$

Using the inequality

$$
\frac{\mathrm{Vol}_{g} A_{g}}{\operatorname{Vol}_{g} B_{g}} \leq\left(\frac{10 \log \left(g^{1 /(10 \tau)}\right)}{g}\right)^{g / 2}=\left(\frac{\log g}{\tau g}\right)^{g / 2}
$$

(see [Bá-Fü] or $[\mathrm{Gl}]$ ), we bound the volume of the convex polytope $A_{g}$ :

$$
\frac{\mathrm{Vol}_{g} A_{g}}{\operatorname{Vol}_{g} \Pi_{g}}<\left(\frac{\pi e \log g}{\tau g}\right)^{g / 2} .
$$

Thus,

for $d$ sufficiently large.

$$
\frac{\operatorname{Vol}_{g} \mathcal{O}_{G}(A)}{\operatorname{Vol}_{g} \mathcal{O}_{G}(P)}<\left(d^{1 / 2-6 \tau}\right)^{-g}
$$

If $g<d^{1-10 \tau}$, then we bound $\operatorname{Vol}_{g} \mathcal{O}_{G}(A)$ from above trivially:

$$
\begin{aligned}
\operatorname{Vol}_{g} \mathcal{O}_{G}(A) & \leq \operatorname{Vol}_{g} \mathcal{O}_{G}(P) \\
& =\operatorname{Vol}_{g} \mathcal{O}_{G}(P)\left(d^{1 / 2-6 \tau}\right)^{-g}\left(d^{1 / 2-6 \tau}\right)^{g} \\
& <\operatorname{Vol}_{g} \mathcal{O}_{G}(P)\left(d^{1 / 2-6 \tau}\right)^{-g} \exp \left(d^{1-9 \tau}\right) .
\end{aligned}
$$

Thus for all $G \subset\{1, \ldots, d\}$ we have the upper bound

$$
\begin{aligned}
\operatorname{Vol}_{g} \mathcal{O}_{G}(A) & <\operatorname{Vol}_{g} \mathcal{O}_{G}(P)\left(d^{1 / 2-6 \tau}\right)^{-g} \exp \left(d^{1-9 \tau}\right) \\
& =\exp \left(d^{1-9 \tau}\right)\left(d^{1 / 2-6 \tau}\right)^{-g} \prod_{j \in G} u_{j} .
\end{aligned}
$$

Now Lemma 1 follows from Lemma 0.

For a vector $w=\left(w_{1}, \ldots, w_{d}\right) \in \mathbb{C}^{d}$ define

$$
S_{k}(w)=\sum_{j=1}^{d} w_{j}^{k}
$$

and write $\|w\|=\max _{1 \leq j \leq d}\left|w_{j}\right|$ for the $l_{\infty}$ norm. We denote by $\Re z$ and $\Im z$ the real and imaginary part of $z$ respectively.

Lemma 2. Let $W$ be a subset of $\mathbb{C}^{d}$ such that $\|w\| \leq \exp \left(\frac{\log d}{10 d}\right)$ for all $w \in W$ and

$$
\max _{1 \leq k \leq d}\left|\Re\left(S_{k}(u)-S_{k}(v)\right)\right| / k \geq d^{-1 / 5}
$$


for any two distinct $u, v \in W$. Then

$$
\text { card } W<\exp \left(d^{8 / 9}\right) \text {. }
$$

Proof. For a complex number $z=a+i b$ we define

$$
\widetilde{z}=d^{-3}\left(\left[|a| d^{3}\right] \operatorname{sgn} a+i\left[|b| d^{3}\right] \operatorname{sgn} b\right) .
$$

Then $|\widetilde{z}| \leq|z|$ and

$$
|z-\widetilde{z}|=d^{-3}\left(\left\{|a| d^{3}\right\}^{2}+\left\{|b| d^{3}\right\}^{2}\right)^{1 / 2}<\sqrt{2} d^{-3} .
$$

If $w=\left(w_{1}, \ldots, w_{d}\right)$, then write $\widetilde{w}$ for the vector with coordinates $\widetilde{w}_{j}$. For $w \in W$ and $1 \leq k \leq d$ we have

$$
\begin{aligned}
\left|S_{k}(\widetilde{w})-S_{k}(w)\right| & =\left|\sum_{j=1}^{d}\left(\widetilde{w}_{j}^{k}-w_{j}^{k}\right)\right| \leq d \max _{1 \leq j \leq d}\left|\widetilde{w}_{j}^{k}-w_{j}^{k}\right| \\
& <\sqrt{2} d^{-2} k \exp \left(\frac{(k-1) \log d}{10 d}\right)<2 k d^{-19 / 10} .
\end{aligned}
$$

Hence there exists a number $K=K(u, v), 1 \leq K \leq d$, such that

$$
\begin{aligned}
\left|\Re\left(S_{K}(\widetilde{u})-S_{K}(\widetilde{v})\right)\right| & >\left|\Re\left(S_{K}(u)-S_{K}(v)\right)\right|-4 K d^{-19 / 10} \\
& \geq K d^{-1 / 5}-4 K d^{-19 / 10}>K d^{-1 / 5} / 2 .
\end{aligned}
$$

Let $D$ be the set of vectors

$$
\left(2 d^{1 / 5} \Re S_{1}(\widetilde{w}), \ldots, 2 k^{-1} d^{1 / 5} \Re S_{k}(\widetilde{w}), \ldots, 2 d^{-4 / 5} \Re S_{d}(\widetilde{w})\right)
$$

in $\mathbb{R}^{d}$ where $w \in W$. By the above we see that the map $W \rightarrow D$ is injective and that the distance between any two elements of $D$ is at least 1 . Let $A$ be the convex hull of the vectors

$$
\left(2 d^{6 / 5} \Re y, \ldots, 2 k^{-1} d^{6 / 5} \Re y^{k}, \ldots, 2 d^{1 / 5} \Re y^{d}\right) .
$$

Here $|y| \leq \exp \left(\frac{\log d}{10 d}\right)$ and $d^{3} y \in \mathbb{Z}[i]$, i.e. $y$ runs over the set of all possible points $\widetilde{w}_{j}$. In particular, each vector in $D$ is the arithmetic mean of some $d$ of the above vectors. Hence $D$ is a subset of $A$. Clearly, the number of vertices in the polytope $A$ is bounded above by the number of Gauss' integers in the circle $|y| \leq d^{3} \exp \left(\frac{\log d}{10 d}\right)$. An upper bound for these is obtained by counting the number of unit squares with center at Gauss' integer:

$$
\pi\left(\sqrt{2}+d^{3} \exp \left(\frac{\log d}{10 d}\right)\right)^{2}<4 d^{6} .
$$

Moreover, $A$ is contained in the parallelepiped

$$
P=\prod_{j=1}^{d}\left[-u_{j} / 2 ; u_{j} / 2\right] \quad \text { with } \quad u_{j}=4 j^{-1} d^{6 / 5} \exp \left(\frac{j \log d}{10 d}\right) .
$$


Taking $\tau=1 / 75$ we now apply Lemma 1 :

$$
\operatorname{card} D<\exp \left(d^{22 / 25}\right) \prod_{j=1}^{d}\left(1+4 j^{-1} d^{39 / 50} \exp \left(\frac{j \log d}{10 d}\right)\right) .
$$

Since $1+x<e^{x}, x>0$, we bound the last product by

$$
\exp \left(\sum_{j=1}^{d} 4 j^{-1} d^{39 / 50} \exp \left(\frac{j \log d}{10 d}\right)\right)<\exp \left(5 d^{22 / 25} \log d\right) \text {. }
$$

Thus,

$$
\operatorname{card} W=\operatorname{card} D<\exp \left(d^{22 / 25}(1+5 \log d)\right)<\exp \left(d^{8 / 9}\right) .
$$

Lemma 3. Let $n \in \mathbb{N}$ and let $N_{u} \in \mathbb{N}, r_{u} \in \mathbb{N} \cup\{0\}, b_{u} \in \mathbb{R}, b_{u} \geq 1$, for $u=0,1,2, \ldots$ If $N_{u} \leq b_{u} n$ and $\sum_{u \geq 0} r_{u}=n$, then

$$
\prod_{u \geq 0}\left(\begin{array}{c}
N_{u}-1+r_{u} \\
r_{u}
\end{array}\right) \leq n^{n} \prod_{u \geq 0} b_{u}^{r_{u}}
$$

and

$$
\prod_{u \geq 0}\left(\begin{array}{c}
N_{u}-1+r_{u} \\
r_{u}
\end{array}\right) \leq\left(22\left(1+\frac{B \log n}{n}\right)\right)^{n} \prod_{u \geq 0} b_{u}^{r_{u}},
$$

where $B=\sum_{u \geq 0} r_{u} u$.

Proof. Since

$$
\left(\begin{array}{c}
N_{u}-1+r_{u} \\
r_{u}
\end{array}\right)=\frac{N_{u}+r_{u}-1}{r_{u}} \cdot \frac{N_{u}+r_{u}-2}{r_{u}-1} \cdot \ldots \cdot \frac{N_{u}}{1} \leq N_{u}^{r_{u}},
$$

we get (6) immediately.

In order to prove (7) we assume that $n \geq 23$ and $B>0$. Indeed, if $n \leq 22$ then inequality (7) follows from (6). If $B=0$, then $r_{0}=n$ and using $n !>(n / e)^{n}$ we find that

$$
\begin{aligned}
\prod_{u \geq 0}\left(\begin{array}{c}
N_{u}-1+r_{u} \\
r_{u}
\end{array}\right) & =\left(\begin{array}{c}
N_{0}-1+r_{0} \\
r_{0}
\end{array}\right) \leq \frac{\left(b_{0} n+n\right)^{n}}{n !} \\
& <\left(e\left(b_{0}+1\right)\right)^{n} \leq\left(2 e b_{0}\right)^{n}<\left(22 b_{0}\right)^{n} .
\end{aligned}
$$

Put now $U=\left[\frac{B \log n}{n}\right]$. We have

$$
B \geq \sum_{u \geq U+1} r_{u} u \geq(U+1) \sum_{u \geq U+1} r_{u} .
$$

Thus,

$$
\sum_{0 \leq u \leq U} r_{u}=n-\sum_{u \geq U+1} r_{u} \geq n-\frac{B}{U+1}>n\left(1-\frac{1}{\log n}\right) .
$$


Since the function $x \log x$ is convex, we obtain

$$
\sum_{0 \leq u \leq U} r_{u} \log r_{u}>n\left(1-\frac{1}{\log n}\right) \log \left(\frac{n(1-1 / \log n)}{U+1}\right) .
$$

It follows that

$$
\text { (8) } \begin{aligned}
\prod_{u \geq 0} r_{u}^{r_{u}} & \geq \prod_{0 \leq u \leq U} r_{u}^{r_{u}}>\left(n\left(1-\frac{1}{\log n}\right)\right)^{n(1-1 / \log n)}\left(\frac{B \log n}{n}+1\right)^{-n} \\
& \geq n^{n}\left(\frac{1}{e}\left(1-\frac{1}{\log 23}\right)\right)^{n}\left(\frac{B \log n}{n}+1\right)^{-n} .
\end{aligned}
$$

Utilizing the inequalities $r_{u} \leq n$ and $r_{u} ! \geq\left(r_{u} / e\right)^{r_{u}}$ we obtain

$$
\left(\begin{array}{c}
N_{u}-1+r_{u} \\
r_{u}
\end{array}\right) \leq \frac{\left(N_{u}+n\right)^{r_{u}}}{r_{u} !} \leq\left(\frac{e\left(b_{u}+1\right) n}{r_{u}}\right)^{r_{u}} \leq\left(\frac{2 e b_{u} n}{r_{u}}\right)^{r_{u}} .
$$

Thus, applying (8), we see that the left hand side of (7) does not exceed

$$
\begin{aligned}
\prod_{u \geq 0}\left(2 e b_{u} n\right)^{r_{u}} r_{u}^{-r_{u}} & <\left(\frac{2 e^{2}}{1-1 / \log 23}\right)^{n}\left(\frac{B \log n}{n}+1\right)^{n} \prod_{u \geq 0} b_{u}^{r_{u}} \\
& <\left(22\left(1+\frac{B \log n}{n}\right)\right)^{n} \prod_{u \geq 0} b_{u}^{r_{u}} .
\end{aligned}
$$

3. Sketch of proof. For each polynomial $f$ in $N(d, T)$ we define a vector with nonnegative entries in the following way. Suppose first that the leading coefficient $a_{0}$ is in the range $1 \leq a_{0}<d^{1 / 5} / 2$. Then the vector has the form

$$
\left(a_{0}, q_{1}, q_{2}, n_{l}, s_{l}, n_{l+1}, s_{l+1}, \ldots\right) .
$$

Here $l=\left[\frac{\log d}{10}\right], n_{u}$ is the number of zeros of $f$ lying in

$$
K_{u}=\left\{z \in \mathbb{C}: \exp \left(\frac{u}{d}\right)<|z| \leq \exp \left(\frac{u+1}{d}\right), \Im z>0\right\},
$$

$s_{u}$ is the number of zeros of $f$ lying in

$$
L_{u}=\left[-\exp \left(\frac{u+1}{d}\right) ;-\exp \left(\frac{u}{d}\right)\right) \cup\left(\exp \left(\frac{u}{d}\right) ; \exp \left(\frac{u+1}{d}\right)\right],
$$

$q_{1}=\left[d \log \left|\Lambda_{1}\right|\right], q_{2}=\left[d \log \left|\Lambda_{2}\right|\right]$ where $\Lambda_{1}$ and $\Lambda_{2}$ are the products of zeros of $f$ lying in $\bigcup_{u \geq l} K_{u}$ and $\bigcup_{u \geq l} L_{u}$ respectively.

If however $a_{0} \geq d^{1 / 5} / 2$, then the vector has the form

$$
\left(a_{0}, q_{3}, q_{4}, q_{5}, q_{6}, n_{0}, s_{0}, m_{0}, r_{0}, n_{1}, s_{1}, m_{1}, r_{1}, \ldots\right) .
$$

Here $a_{0}, n_{u}, s_{u}$ are as above, $m_{u}$ is the number of zeros of $f$ lying in

$$
M_{u}=\left\{z \in \mathbb{C}: \exp \left(-\frac{u+1}{d}\right)<|z| \leq \exp \left(-\frac{u}{d}\right), \Im z>0\right\},
$$


$r_{u}$ is the number of zeros of $f$ lying in

$$
R_{u}=\left[-\exp \left(-\frac{u}{d}\right) ;-\exp \left(-\frac{u+1}{d}\right)\right) \cup\left(\exp \left(-\frac{u+1}{d}\right) ; \exp \left(-\frac{u}{d}\right)\right],
$$

$q_{k}=\left[(-1)^{k+1} d \log \left|\Lambda_{k}\right|\right], k=3, \ldots, 6$, where $\Lambda_{3}, \ldots, \Lambda_{6}$ are the products of the zeros of $f$ lying in $\{|z|>1, \Im z>0\},\{|z| \leq 1, \Im z>0\},(-\infty ;-1) \cup$ $(1 ; \infty)$ and $[-1,1]$ respectively.

Now we prove that the number of all different vectors which are defined as above for the polynomials in $N(d, T), T>\exp \left((\log d)^{-3}\right)$, is less than

$$
T^{c_{5} \sqrt{d}(\log d)^{3 / 2}} \text {. }
$$

Indeed, notice first that the number of different vectors $\left(a_{0}, q_{1}, \ldots, q_{6}\right)$ is at most

$$
T(d \log T+1)^{6}<T(d T)^{6}<T^{6(\log d)^{4}+7} .
$$

Put $x_{u}=\sum_{k \geq u} n_{k}, u=1,2, \ldots$ Then the values $n_{1}, n_{2}, \ldots$ are determined uniquely by $x_{1}, x_{2}, \ldots$ We also have $x_{1} \geq x_{2} \geq \ldots$ Since all $x_{k}$ are nonnegative integers and

$$
d \log \left|\Lambda_{3}\right| \geq \sum_{u \geq 1} \sum_{z \in K_{u}} \log |z| \geq \sum_{u \geq 1} u n_{u}=\sum_{u \geq 1} x_{u}
$$

the number of different vectors $\left(n_{1}, n_{2}, \ldots\right)$ is bounded from above by

$$
\sum_{q=0}^{q_{3}} p(q) \leq\left(q_{3}+1\right) p\left(q_{3}\right)
$$

where $p(q)$ is the number of partitions of $q$. The well known asymptotic formula for the number of partitions (see, e.g., $[\mathrm{An}]$ )

$$
p(q) \sim \frac{\exp (\pi \sqrt{2 q / 3})}{4 \sqrt{3} q}
$$

implies that the number of different vectors $\left(n_{1}, n_{2}, \ldots\right)$ does not exceed $c_{6} \exp \left(c_{7} \sqrt{q_{3}}\right)$. Similarly, the number of different vectors $\left(s_{1}, s_{2}, \ldots\right)$, $\left(m_{1}, m_{2}, \ldots\right),\left(r_{1}, r_{2}, \ldots\right)$ is bounded above by $c_{6} \exp \left(c_{7} \sqrt{q_{5}}\right), c_{6} \exp \left(c_{7} \sqrt{q_{4}}\right)$ and $c_{6} \exp \left(c_{7} \sqrt{q_{6}}\right)$ respectively. Since the number of different $\left(n_{0}, s_{0}, m_{0}, r_{0}\right)$ is at most $d^{4}$, bounding $\left|\Lambda_{1}\right|,\left|\Lambda_{2}\right|,\left|\Lambda_{3}\right|,\left|\Lambda_{4}\right|^{-1},\left|\Lambda_{5}\right|,\left|\Lambda_{6}\right|^{-1}$ from above by $T$, we estimate the number of all different vectors by

$$
T^{6(\log d)^{4}+7} d^{4} c_{6}^{4} \exp \left(4 c_{7} \sqrt{d \log T}\right)<T^{c_{5} \sqrt{d}(\log d)^{3 / 2}} .
$$

We see that the number of different vectors is less than $T^{c_{8} d \log \log d / \log d}$. Therefore we only need to prove the upper estimates for the cardinality (ii) and (iii) for the polynomials in $N(d, T), T>\exp \left((\log d)^{-3}\right)$, corresponding to the fixed vector $\left(a_{0}, q_{1}, q_{2}, n_{l}, s_{l}, \ldots\right)$ or $\left(a_{0}, q_{3}, q_{4}, q_{5}, q_{6}, n_{0}, s_{0}, m_{0}, r_{0}, \ldots\right)$. 
For the polynomial

$$
f=a_{0} x^{d}+a_{1} x^{d-1}+\ldots+a_{d}=a_{0}\left(x-\alpha_{1}\right)\left(x-\alpha_{2}\right) \ldots\left(x-\alpha_{d}\right)
$$

we define

$$
S_{k}=S_{k}(f)=\sum_{j=1}^{d} \alpha_{j}^{k} .
$$

By the Newton identities we have

$$
a_{0} S_{k}+a_{1} S_{k-1}+\ldots+a_{k-1} S_{1}+k a_{k}=0 .
$$

Suppose that $f$ and

$$
g=a_{0} x^{d}+h_{1} x^{d-1}+\ldots+h_{d}=a_{0}\left(x-\beta_{1}\right)\left(x-\beta_{2}\right) \ldots\left(x-\beta_{d}\right)
$$

are two distinct polynomials with the same leading coefficient $a_{0}$. There exists a positive integer $k \leq d$ such that $h_{1}=a_{1}, \ldots, h_{k-1}=a_{k-1}$, but $h_{k} \neq a_{k}$. Then $S_{1}(f)=S_{1}(g), \ldots, S_{k-1}(f)=S_{k-1}(g)$, but $S_{k}(f) \neq S_{k}(g)$. From (10) we deduce that

$$
\left|S_{k}(f)-S_{k}(g)\right|=\left|a_{k}-h_{k}\right| \frac{k}{a_{0}} \geq \frac{k}{a_{0}} .
$$

Consider now the polynomials in $N(d, T)$ corresponding to the fixed vector $\left(a_{0}, q_{1}, q_{2}, n_{l}, s_{l}, n_{l+1}, s_{l+1}, \ldots\right)$ where $1 \leq a_{0}<d^{1 / 5} / 2$. Put

$$
n=\sum_{u \geq l} n_{u}, \quad s=\sum_{u \geq l} s_{u} .
$$

We can assume that $\alpha_{1}=\bar{\alpha}_{n+1}, \ldots, \alpha_{n}=\bar{\alpha}_{2 n}$ lie in $\bigcup_{u>l} K_{u}$ and that $\alpha_{2 n+1}, \ldots, \alpha_{2 n+s} \in \bigcup_{u \geq l} L_{u}$ (and similarly for the roots $\beta_{j}$ of $g$ ). From (11) we find that either

$$
\left|\sum_{j=2 n+s+1}^{d}\left(\alpha_{j}^{k}-\beta_{j}^{k}\right)\right| \geq \frac{k}{2 a_{0}}
$$

or

$$
\left|\sum_{j=1}^{2 n+s}\left(\alpha_{j}^{k}-\beta_{j}^{k}\right)\right| \geq \frac{k}{2 a_{0}} .
$$

Below we will argue as follows. All $K_{u}$ and $L_{u}$ will be covered by disjoint squares and intervals respectively (see Section 4 ). The crucial step is to estimate the number of ways to distribute $n+s$ roots into corresponding squares and intervals. We will show that for each distribution where each square and interval contains the same number of $\alpha_{j}$ 's and $\beta_{j}$ 's, $1 \leq j \leq$ $2 n+s$, the inequality opposite to (13) holds. Thus, inequality (12) holds. We see that the $l_{\infty}$ norm of the vectors $\left(0,0, \ldots, 0, \alpha_{2 n+s+1}, \ldots, \alpha_{d}\right)$ and 
$\left(0,0, \ldots, 0, \beta_{2 n+s+1}, \ldots, \beta_{d}\right)$ is bounded above by $\exp \left(\frac{\log d}{10 d}\right)$. Since

$$
\left|\Re \sum_{j=2 n+s+1}^{d}\left(\alpha_{j}^{k}-\beta_{j}^{k}\right)\right|=\left|\sum_{j=2 n+s+1}^{d}\left(\alpha_{j}^{k}-\beta_{j}^{k}\right)\right| \geq \frac{k}{2 a_{0}}>k d^{-1 / 5},
$$

we can apply Lemma 2. For any way to distribute roots the number of distinct polynomials corresponding to this way is bounded above by

$$
\exp \left(d^{8 / 9}\right)<\exp \left(d^{8 / 9}(\log T)(\log d)^{3}\right)<T^{d^{9 / 10}} .
$$

4. Polynomials with small leading coefficient. For any nonnegative integer $u$ we cover $K_{u}$ by disjoint squares with side of length

$$
\frac{\exp (-(u+1)(d-1) / d)}{7 \sqrt{2} n a_{0}} .
$$

If $\alpha_{j}$ and $\beta_{j}$ belong to the same square, then

$$
\begin{aligned}
\left|\alpha_{j}^{k}-\beta_{j}^{k}\right| & =\left|\alpha_{j}-\beta_{j}\right| \cdot\left|\alpha_{j}^{k-1}+\alpha_{j}^{k-2} \beta_{j}+\ldots+\beta_{j}^{k-1}\right| \\
& \leq \frac{\exp (-(u+1)(d-1) / d)}{7 n a_{0}} k \exp \left(\frac{(u+1)(k-1)}{d}\right) \leq \frac{k}{7 n a_{0}}
\end{aligned}
$$

for $k \leq d$. Therefore, if each square contains the same number of $\alpha_{j}$ 's and $\beta_{j}$ 's, then

We also have

$$
\left|\sum_{j=1}^{n}\left(\alpha_{j}^{k}-\beta_{j}^{k}\right)\right| \leq \frac{k}{7 a_{0}}
$$

$$
\left|\sum_{j=n+1}^{2 n}\left(\alpha_{j}^{k}-\beta_{j}^{k}\right)\right|=\left|\sum_{j=n+1}^{2 n}\left(\bar{\alpha}_{j}^{k}-\bar{\beta}_{j}^{k}\right)\right|=\left|\sum_{j=1}^{n}\left(\alpha_{j}^{k}-\beta_{j}^{k}\right)\right| \leq \frac{k}{7 a_{0}} .
$$

Similarly, cover $L_{u}$ by disjoint intervals of length

$$
\frac{\exp (-(u+1)(d-1) / d)}{7 s a_{0}} \text {. }
$$

If each interval contains the same number of $\alpha_{j}$ 's and $\beta_{j}$ 's, we have

$$
\left|\sum_{j=2 n+1}^{2 n+s}\left(\alpha_{j}^{k}-\beta_{j}^{k}\right)\right| \leq \frac{k}{7 a_{0}} \text {. }
$$

Thus,

which contradicts (13).

$$
\left|\sum_{j=1}^{2 n+s}\left(\alpha_{j}^{k}-\beta_{j}^{k}\right)\right| \leq \frac{3 k}{7 a_{0}}
$$

Clearly, the number of polynomials with different distribution of roots is bounded above by the number of ways to distribute roots into respective 
squares and intervals. More precisely, this is the number of ways to put $n_{u}$, $u=l, l+1, \ldots$, roots into $N_{u}$ squares times the number of ways to put $s_{u}$, $u=l, l+1, \ldots$, roots into intervals. The number of squares $N_{u}$ with side length (15) which cover $K_{u}$ is bounded from above by

$$
\begin{aligned}
& \frac{\pi}{2} 98 n^{2} a_{0}^{2} \exp \left(\frac{2(u+1)(d-1)}{d}\right)\left(\left(\exp \left(\frac{u+1}{d}\right)\right.\right. \\
& \left.\left.+\frac{\exp (-(u+1)(d-1) / d)}{7 n a_{0}}\right)^{2}-\left(\exp \left(\frac{u}{d}\right)-\frac{\exp (-(u+1)(d-1) / d)}{7 n a_{0}}\right)^{2}\right) .
\end{aligned}
$$

By a short computation we see that this is bounded by $c_{9} n a_{0}^{2} \exp (2 u)$. The number of ways to put $n_{u}$ roots into $N_{u}$ squares equals $\left(\begin{array}{c}N_{u}+n_{u}-1 \\ n_{u}\end{array}\right)$.

In order to estimate the product

$$
\prod_{u \geq l}\left(\begin{array}{c}
N_{u}+n_{u}-1 \\
n_{u}
\end{array}\right)
$$

we apply Lemma 3 with $b_{u}=c_{9} a_{0}^{2} \exp (2 u)$ and $B=q_{1}$. Indeed, the inequality

$$
\sum_{u \geq l} u n_{u} \leq d \log \left|\Lambda_{1}\right|<q_{1}+1
$$

implies that

$$
\sum_{u \geq l} u n_{u} \leq q_{1}
$$

If $n \leq q_{1}(\log d)^{-2}$, then by (6) we have

$$
\begin{aligned}
\prod_{u \geq l}\left(\begin{array}{c}
N_{u}+n_{u}-1 \\
n_{u}
\end{array}\right) & \leq n^{n} \prod_{u \geq l}\left(c_{9} a_{0}^{2}\right)^{n_{u}} \exp \left(2 u n_{u}\right) \\
& \leq c_{9}^{n} a_{0}^{2 n} \exp \left(q_{1}(\log d)^{-1}+2 q_{1}\right) .
\end{aligned}
$$

If $n>q_{1}(\log d)^{-2}$, then by (7) we find

$$
\begin{aligned}
\prod_{u \geq l}\left(\begin{array}{c}
N_{u}+n_{u}-1 \\
n_{u}
\end{array}\right) & \leq\left(22\left(1+\frac{q_{1} \log n}{n}\right)\right)^{n} c_{9}^{n} a_{0}^{2 n} \exp \left(2 q_{1}\right) \\
& <\left(23(\log d)^{3}\right)^{n} c_{9}^{n} a_{0}^{2 n} \exp \left(2 q_{1}\right) \\
& <c_{10}^{n} a_{0}^{2 n} \exp \left(2 q_{1}+3 n \log \log d\right) .
\end{aligned}
$$

Since

$$
q_{1} \geq \sum_{u \geq l} u n_{u} \geq n l=n\left[\frac{\log d}{10}\right]
$$


in both cases (17) and (18) we have

$$
\prod_{u \geq l}\left(\begin{array}{c}
N_{u}+n_{u}-1 \\
n_{u}
\end{array}\right) \leq a_{0}^{2 n} \exp \left(2 q_{1}+31 q_{1} \log \log d / \log d\right) .
$$

Analogously, the number of intervals of length (16) which cover $L_{u}$ is bounded above by

$$
\begin{aligned}
14 s a_{0} \exp \left(\frac{(u+1)(d-1)}{d}\right) & \left(\exp \left(\frac{u+1}{d}\right)-\exp \left(\frac{u}{d}\right)\right. \\
+ & \left.\frac{\exp (-(u+1)(d-1) / d)}{3 s a_{0}}\right)<c_{11} a_{0} \exp (u) .
\end{aligned}
$$

Since $\sum_{u>l} u s_{u} \leq q_{2}$, the number of ways to distribute $s_{u}, u=l, l+1, \ldots$, roots into respective intervals does not exceed

$$
\prod_{u \geq l}\left(c_{11} a_{0} \exp (u)\right)^{s_{u}} \leq c_{11}^{s} a_{0}^{s} \exp \left(q_{2}\right) .
$$

Using the inequality

$$
s \leq \frac{q_{2}}{l} \leq \frac{11 q_{2}}{\log d}
$$

we further bound (20) from above by $a_{0}^{s} \exp \left(q_{2}+q_{2} \log \log d / \log d\right)$. Since $2 n+s \leq d$ and $2 q_{1}+q_{2} \leq d \log \left(T / a_{0}\right)$, combining this inequality with (19) we estimate the number of ways to distribute roots into respective squares and intervals by

$$
\begin{aligned}
a_{0}^{2 n+s} \exp \left(2 q_{1}+q_{2}+15.5\left(2 q_{1}+q_{2}\right) \log \log d / \log d\right) \\
<T^{d(1+15.5 \log \log d / \log d)} .
\end{aligned}
$$

5. Polynomials with large leading coefficient. In this section we are left with the case $a_{0} \geq d^{1 / 5} / 2$. We need an upper bound for the number of polynomials corresponding to the vector $\left(a_{0}, q_{3}, q_{4}, q_{5}, q_{6}, n_{0}, s_{0}, m_{0}, r_{0}, n_{1}\right.$, $\left.s_{1}, m_{1}, r_{1}, \ldots\right)$. Put now

$$
n=\sum_{u \geq 0} n_{u}, \quad s=\sum_{u \geq 0} s_{u}, \quad m=\sum_{u \geq 0} m_{u}, \quad r=\sum_{u \geq 0} r_{u} .
$$

We enumerate the roots of two distinct polynomials $f$ and $g$ as follows. Let the first $n$ roots of $f$ (and $g$ ) lie outside the unit circle in the upper halfplane, then their $n$ complex conjugates, then $s$ real roots in $(-\infty ;-1) \cup(1 ; \infty)$, then $m$ complex roots in the unit circle in the upper halfplane, then their $m$ complex conjugates, and finally $r$ real roots in $[-1 ; 1]$. As in Section 3 we obtain 


$$
\begin{aligned}
\frac{k}{a_{0}} \leq\left|\sum_{j=1}^{d}\left(\alpha_{j}^{k}-\beta_{j}^{k}\right)\right| \leq & 2\left|\sum_{j=1}^{n}\left(\alpha_{j}^{k}-\beta_{j}^{k}\right)\right|+\left|\sum_{j=2 n+1}^{2 n+s}\left(\alpha_{j}^{k}-\beta_{j}^{k}\right)\right| \\
& +2\left|\sum_{j=2 n+s+1}^{2 n+s+m}\left(\alpha_{j}^{k}-\beta_{j}^{k}\right)\right|+\left|\sum_{j=2 n+s+2 m+1}^{d}\left(\alpha_{j}^{k}-\beta_{j}^{k}\right)\right| .
\end{aligned}
$$

It follows that one of the four sums is at least $k /\left(7 a_{0}\right)$. Again the crucial step is to estimate the number of ways to distribute $n+s+m+r$ roots into corresponding squares and intervals. Suppose first that

$$
\left|\sum_{j=1}^{n}\left(\alpha_{j}^{k}-\beta_{j}^{k}\right)\right|>\frac{k}{7 a_{0}} .
$$

As in Section 4 we cover $K_{u}$ by disjoint squares with side length (15). If each square contains the same number of $\alpha_{j}$ 's and $\beta_{j}$ 's, $1 \leq j \leq n$, then the inequality opposite to the above holds. In a similar manner to that in Section 4 we deduce the upper estimate for the number of ways to distribute $n$ roots into squares. Instead of (17) and (18) we have the upper bounds $c_{9}^{n} a_{0}^{2 n} \exp \left(2 q_{3}+q_{3}(\log d)^{-1}\right)$ and $c_{10}^{n} a_{0}^{2 n} \exp \left(2 q_{3}+3 n \log \log d\right)$ respectively. Combining both cases we obtain the upper estimate for the number of ways to distribute $n$ roots:

$$
a_{0}^{2 n} \exp \left(2 q_{3}+q_{3}(\log d)^{-1}+3 n \log \log d+n c_{12}\right) .
$$

Suppose now that

$$
\left|\sum_{j=2 n+1}^{2 n+s}\left(\alpha_{j}^{k}-\beta_{j}^{k}\right)\right|>\frac{k}{7 a_{0}} .
$$

The number of ways to distribute $s$ roots is bounded from above analogously to (20) by

$$
a_{0}^{s} \exp \left(q_{5}+s c_{13}\right)
$$

Next we suppose that the third sum is large:

$$
\left|\sum_{j=2 n+s+1}^{2 n+s+m}\left(\alpha_{j}^{k}-\beta_{j}^{k}\right)\right|>\frac{k}{7 a_{0}} .
$$

Cover $M_{u}$ by disjoint squares with side length $1 /\left(7 \sqrt{2} m a_{0}\right)$. If $\alpha_{j}$ and $\beta_{j}$ belong to the same square, then

$$
\left|\alpha_{j}^{k}-\beta_{j}^{k}\right| \leq\left|\alpha_{j}-\beta_{j}\right| k \leq \frac{k}{7 m a_{0}} .
$$


Thus, if each square contains the same number of $\alpha_{j}$ 's and $\beta_{j}$ 's, then

$$
\left|\sum_{j=2 n+s+1}^{2 n+s+m}\left(\alpha_{j}^{k}-\beta_{j}^{k}\right)\right| \leq \frac{k}{7 a_{0}} .
$$

Therefore, we need an upper estimate for the number of ways to put $m_{u}$ roots into respective squares. The number of squares $S_{u}$ which cover $M_{u}$ is bounded from above by

$$
\frac{\pi}{2} 98 m^{2} a_{0}^{2}\left(\left(\exp \left(-\frac{u}{d}\right)+\frac{1}{7 m a_{0}}\right)^{2}-\left(\exp \left(-\frac{u+1}{d}\right)-\frac{1}{7 m a_{0}}\right)^{2}\right)
$$

for $u+1 \leq d \log \left(7 m a_{0}\right)$. This expression is less than $c_{14} m a_{0}^{2}$. If however $u+1>d \log \left(7 m a_{0}\right)$, then

$$
\exp \left(-\frac{u}{d}\right)<\frac{\exp (1 / d)}{7 m a_{0}}<\frac{1}{6 m a_{0}}
$$

and $M_{u}$ can be covered by 4 squares. Thus, our upper bound $S_{u}<c_{14} m a_{0}^{2}$ also holds.

If $m \leq q_{4}(\log d)^{-2}$, then analogously to (17), by (6) with $b_{u}=c_{14} a_{0}^{2}$, $N_{u}=S_{u}, r_{u}=m_{u}, n=m$ we obtain the upper bound

$$
\prod_{u \geq 0}\left(\begin{array}{c}
S_{u}-1+m_{u} \\
m_{u}
\end{array}\right) \leq m^{m} \prod_{u \geq 0}\left(c_{14} a_{0}^{2}\right)^{m_{u}} \leq c_{14}^{m} a_{0}^{2 m} \exp \left(q_{4} / \log d\right) .
$$

If $m>q_{4}(\log d)^{-2}$, then analogously to (18), by (7) with $B=q_{4}$ we obtain the upper bound

$$
\begin{aligned}
\prod_{u \geq 0}\left(\begin{array}{c}
S_{u}-1+m_{u} \\
m_{u}
\end{array}\right) & \leq\left(22\left(1+\frac{q_{4} \log m}{m}\right)\right)^{m} \prod_{u \geq 0}\left(c_{14} a_{0}^{2}\right)^{m_{u}} \\
& \leq\left(23(\log d)^{3}\right)^{m} c_{14}^{m} a_{0}^{2 m} \leq c_{15}^{m} a_{0}^{2 m} \exp (3 m \log \log d) .
\end{aligned}
$$

Combining these two upper bounds we finally estimate the number of ways to distribute $m$ roots by

$$
a_{0}^{2 m} \exp \left(q_{4}(\log d)^{-1}+3 m \log \log d+m c_{16}\right) .
$$

Finally, suppose that

$$
\left|\sum_{j=2 n+s+2 m+1}^{d}\left(\alpha_{j}^{k}-\beta_{j}^{k}\right)\right|>\frac{k}{7 a_{0}} .
$$

We cover $R_{u}$ by disjoint intervals of length $1 /\left(7 r a_{0}\right)$. The number of intervals which cover $R_{u}$ is bounded above by $c_{17} a_{0}$. Hence, the number of ways to distribute $r$ roots is bounded above by

$$
\prod_{u \geq 0}\left(c_{17} a_{0}\right)^{r_{u}} \leq a_{0}^{r} \exp \left(r c_{18}\right) .
$$


Combining this estimate with (22)-(24) we bound the number of polynomials corresponding to the fixed vector $\left(a_{0}, q_{3}, q_{4}, q_{5}, q_{6}, n_{0}, s_{0}, m_{0}, r_{0}, n_{1}, s_{1}\right.$, $\left.m_{1}, r_{1}, \ldots\right)$ by the number of ways to distribute $n+s+m+r$ roots into respective squares and intervals:

$$
a_{0}^{2 n+s+2 m+r} \exp \left(2 q_{3}+q_{5}+\left(q_{3}+q_{4}\right)(\log d)^{-1}+3(m+n) \log \log d+c_{19} d\right) \text {. }
$$

Since $2 n+s+2 m+r=d, 2 q_{3}+q_{5} \leq d \log \left(T / a_{0}\right)$ and $q_{4} \leq d \log T$, this is less than

$$
T^{d} \exp \left(\frac{2 d \log T}{\log d}+2 d \log \log d\right) .
$$

We now use the fact that $a_{0}$ is large:

$$
T \geq a_{0} \geq d^{1 / 5} / 2>d^{1 / 6} \text {. }
$$

Hence $\log d<6 \log T$, and (25) is less than

$$
T^{d(1+13 \log \log d / \log d)} \text {. }
$$

Thus, we obtain the upper bound which is less than (21). Because of (9) and (14), we finally have

$$
\operatorname{card} N(d, T)<T^{d(1+16 \log \log d / \log d)} .
$$

This completes the proof of (iii).

6. The number of reciprocal polynomials. To prove (ii) notice first that $T<\theta<2$, hence the polynomials lying in $N(d, T)$ are monic. Our argument is based on the following result due to C. Smyth [Sm]: if the Mahler measure of an integer polynomial $f$ is less than $\theta, \theta^{3}=\theta+1$, then the polynomial is reciprocal, i.e. $f(x) \equiv \operatorname{sgn}\left(a_{d}\right) x^{d} f(1 / x)$. Hence $a_{0}=1=\varrho a_{d}$, $a_{1}=\varrho a_{d-1}, a_{2}=\varrho a_{d-2}, \ldots$ where $\varrho=\operatorname{sgn}\left(a_{d}\right)$. Thus, for two distinct monic reciprocal polynomials $f$ and $g$ inequality (11) holds with an integer $k$ in the range $1 \leq k \leq d / 2$.

Replacing the side length of the square in (15) by

$$
\frac{\exp (-(u+1)(d / 2-1) / d)}{7 \sqrt{2} n}
$$

and the length of the interval in (16) by

$$
\frac{\exp (-(u+1)(d / 2-1) / d)}{7 s}
$$

we can argue as in Section 4. The number of squares in this case is bounded above by $c_{9} n \exp (u)$. The right-hand side of (19) is now $\exp \left(q_{1}+31 q_{1} \times\right.$ $\log \log d / \log d)$. Analogously, the number of intervals which cover $L_{u}$ is now $c_{11} \exp (u / 2)$. Thus, the number of ways to distribute $s_{u}, u=l, l+1, \ldots$, roots into respective intervals does not $\operatorname{exceed} \exp \left(q_{2} / 2+q_{2} \log \log d / \log d\right)$. 
Therefore, the number of ways to distribute roots into squares and intervals is bounded above by

$\exp \left(\left(2 q_{1}+q_{2}\right) / 2+15.5\left(2 q_{1}+q_{2}\right) \log \log d / \log d\right)<T^{d(0.5+15.5 \log \log d / \log d)}$.

Combining this inequality with (9) and (14) in the case (ii) we obtain

$$
\operatorname{card} N(d, T)<T^{d(1 / 2+16 \log \log d / \log d)} .
$$

\section{References}

[An] G. E. Andrews, The Theory of Partitions, Addison-Wesley, Reading, Mass., 1976.

[Bá-Fü] I. Bárány and Z. Füredy, Computing the volume is difficult, Discrete Comput. Geom. 2 (1987), 319-326.

[Bo-Mo] D. W. Boyd and H. L. Montgomery, Cyclotomic partitions, in: Number Theory, R. A. Mollin (ed.), de Gruyter, 1990, 7-25.

[Do] E. Dobrowolski, On a question of Lehmer and the number of irreducible factors of a polynomial, Acta Arith. 34 (1979), 391-401.

[Gl] E. D. Gluskin, Extremal properties of orthogonal parallelepipeds and their applications to the geometry of Banach spaces, Mat. Sb. (N.S.) 136 (178) (1988), 85-96 (in Russian).

[Ko] S. V. Konyagin, On the number of irreducible polynomials with 0, 1 coefficients, Acta Arith., submitted.

[La] E. Landau, Sur quelques théorèmes de M. Petrovic relatifs aux zéros des fonctions analytiques, Bull. Soc. Math. France 33 (1905), 251-261.

[Le] D. H. Lehmer, Factorization of certain cyclotomic functions, Ann. of Math. (2) 34 (1933), 461-479.

[Lo] R. Louboutin, Sur la mesure de Mahler d'un nombre algébrique, C. R. Acad. Sci. Paris Sér. I 296 (1983), 707-708.

[Mi] M. Mignotte, Sur les nombres algébriques de petite mesure, Comité des Travaux Historiques et Scientifiques: Bulletin de la Section des Sciences 3 (1981), 65-80.

[Sm] C. J. Smyth, On the product of the conjugates outside the unit circle of an algebraic integer, Bull. London Math. Soc. 3 (1971), 169-175.

[Ti] V. M. Tikhomirov, Some Questions in Approximation Theory, Izdat. Moskov. Univ., Moscow, 1976 (in Russian).

Department of Mathematics

Vilnius University

Naugarduko 24

2006 Vilnius, Lithuania

E-mail: arturas.dubickas@maf.vu.lt
Department of Mechanics and Mathematics Moscow State University 119899 Moscow, Russia E-mail: kon@nw.math.msu.su

Received on 14.11 .1997

and in revised form on 5.6.1998 